\title{
Baker's yeast $(1 \rightarrow 3)-\beta$-D-glucan Influences Insulin Sensitivity in Mice with Humanized Obese Diabetic Microbiome in High-Fat Diet-Induced Obesity
}

\author{
Kathleen A. J. Mitchelson ${ }^{1}$, Elena de Marco Castro ${ }^{1}$, Cara M. Hueston ${ }^{2}$, Gina M. Lynch ${ }^{1}$, \\ Elaine A. Keogh ${ }^{1}$, Tam T.T. Tran ${ }^{2,3}$, Klara Vlckova ${ }^{2,3}$, Helen M. Roche ${ }^{1,4}$ and \\ Paul W. O'Toole 2,3 \\ ${ }^{1}$ Nutrigenomics Research Group and Institute of Food and Health, University College Dublin, Dublin, Ireland, \\ ${ }^{2}$ APC Microbiome Ireland, University College Cork, Cork, Ireland, \\ ${ }^{3}$ School of Microbiology, University College Cork, Cork, Ireland and \\ ${ }^{4}$ Diabetes Complications Research Centre, University College Dublin, Dublin, Ireland
}

\begin{abstract}
Introduction: $\beta$-glucans are naturally occurring polysaccharides which have isoform specific immunomodulatory and metabolic properties $^{(1)}$. Certain yeast $(1 \rightarrow 3)-\beta$-D-glucan isoforms improve cholesterol $^{(2)}$, glucose ${ }^{(3)}$ and lipid homeostasis ${ }^{(4)}$. Feeding $(1 \rightarrow 3)$ - $\beta$-D-glucan alters the microbiome of high-fat diet (HFD) induced obese (DIO)/type 2 diabetic (T2D) mice ${ }^{(5)}$. Here we investigated the potential impact of baker's yeast $(1 \rightarrow 3)-\beta$-D-glucan in mice humanized with gut microbiomes from either obese healthy versus obese diabetic subjects on immune-metabolism within the context of high-fat feeding.
\end{abstract}

Methods: C57Bl/6J male mice received an antibiotic cocktail of Ampicillin, Metronidazole, Vancomycin, Imipenem and Ciprofloxacin $\mathrm{HCl}$ in their drinking water for 6 weeks to diminish the endogenous gut microbiota. Mice were inoculated with microbiota samples obtained from obese healthy $(\mathrm{OBH})$ or diabetic (OBD) humans twice daily for 3 days by oral dosing. Mice were fed a low-fat diet (LFD) $(10 \% \mathrm{kcal})$ for 4 weeks followed by HFD $(45 \% \mathrm{kcal})$ with/without baker's yeast $(1 \rightarrow 3)-\beta$-D-glucan $(\beta \mathrm{G})$, for 9 weeks. Weight, feed intake, glucose tolerance $(1.5 \mathrm{~g} / \mathrm{kg})$, insulin tolerance $(0.5 \mathrm{U} / \mathrm{kg})$, hepatic and skeletal lipid levels were examined. Tissue specific molecular markers of metabolism and inflammation, and gut microbiome analysis are being determined to compliment the phenotypic data.

Results: OBH mice were more glucose tolerant and insulin sensitive than OBD mice, despite equal weight gain and adipose tissue mass. Fasting HOMA-IR, attributable to higher insulin concentrations, was higher in OBD compared to OBH mice. $\beta \mathrm{G}$ supplementation reduced HOMA-IR in OBD mice $(\mathrm{P}<0.0611)$. Hepatic triacylglycerol (TAG) and cholesterol levels were also higher in OBD mice, which were prevented by $\beta G$ supplementation. Hepatic proteomic, caecal microbiomic and metabolomic analysis is on-going in order to ascertain the impact of the OBD versus $\mathrm{OBH}$ dysbosis with/without $\beta \mathrm{G}$ supplementation with specific attention on immune-metabolism.

\section{Conflict of Interest}

There is no conflict of interest

\section{References}

1. Bohn JA \& BeMIller JN (1995) Carbohydrate Polymers 28, 3-14.

2. Kusmiati F, Rizky Dhewantara X et al.. (2016) Scientia Pharmaceutica 84(1), 153-65.

3. Silva Vde O, Lobato RV et al. (2015) PLoS One 10(8), e0134742.

4. Cao Y, Sun, Y et al.. (2017) Journal of Agricultural and Food Chemistry 65(44), 9665-9674.

5. Cao Y, Zou S et al.. (2016) Molecular Nutrition \& Food Research 60, 2678-2690. 